\title{
ACTIVE REGIONS FOR COLOUR TEXTURE SEGMENTATION INTEGRATING REGION AND BOUNDARY INFORMATION
}

\author{
X. Muñoz, J. Freixenet, X. Cufí and J. Martí \\ Computer Vision and Robotics Group. Institute of Informatics and Applications \\ University of Girona. Campus de Montilivi s/n. 17071. Girona, Spain \\ \{xmunoz, jordif, xcuf, joanm\}@eia.udg.es
}

\begin{abstract}
In this paper a colour texture segmentation method which unifies region and boundary information is proposed. The algorithm uses a coarse detection of the perceptual (colour and texture) edges of the image to adequately place and initialise a set of active regions. Colour texture of regions is modelled by the conjunction of non-parametric techniques of kernel density estimation (which allow to estimate the colour behaviour) and classical co-occurrence matrix based texture features. Therefore, region information is defined and accurate boundary information can be extracted to guide the segmentation process. Regions concurrently compete for the image pixels in order to segment the whole image taking both information sources into account. Furthermore, experimental results are shown which prove the performance of the proposed method.
\end{abstract}

\section{INTRODUCTION}

The aim of colour texture segmentation, the problem considered in this paper, is the domain-independent partition of the image into a set of regions, which are visually distinct and uniform with respect to colour and textural properties. Image segmentation methods are based on two basic properties of the pixels in relation to their local neighbourhood: discontinuity and similarity. Methods which are based on some discontinuity property are called boundarybased methods and their objective is to accurately extract the borders between regions in an image. Whereas, methods based on some similarity property, called region-based methods, try to part the image into a number of regions such that each region has the same properties.

Based on the complementary nature of edge and region information, current trends on image segmentation wage for the integration of both sources in order to obtain better results and to solve the problems that both methods bear when

\footnotetext{
This work was partially developed thanks to the support of the Departament d'Universitats, Recerca i Societat de la Informació de la Generalitat de Catalunya.
}

are used separately. However, the texture is generally forgotten as basic feature in the majority of these proposals [1], although there are some relevant exceptions and this tendency seems to be changing in last years [2].

On other hand, there are two basic properties that can be considered for grouping pixels: colour and texture. Both features are basic in order to define the visual perception [3] and experiments have demonstrated that the inclusion of colour can increase the texture segmentation/classification results without significantly complicating the feature extraction algorithms [4]. Nevertheless, most of the literature deals with segmentation based on either colour or texture, and there is a limited number of systems which consider both properties together.

Moreover, to knowledge of the authors, there is not so far any proposal which integrates region and boundary information sources taking colour and texture properties into account. Hence, in this paper we extend our previous proposal of segmentation technique [5], which integrates region and boundary information for texture segmentation, to carry out the colour texture segmentation. The remainder of this paper is structured as follows: Section 2 describes the proposed region segmentation technique, detailing the placement of starting seeds, the definition of region and boundary information and the growing of active regions. Experimental results proving the validity of our proposal appear in Section 3. Finally, conclusions are given in Section 4.

\section{NEW PROPOSAL BASED ON ACTIVE REGION SEGMENTATION}

The concept of active regions as a way to combine the region and boundary information has been recently introduced. This model is a considerable extension on the active contour model since it incorporates region-based information with the aim of finding a partition where the interior and the exterior of the region preserve the desired image properties.

Our approach uses the perceptual edges of the image to adequately place a set of seeds in order to initialise the ac- 


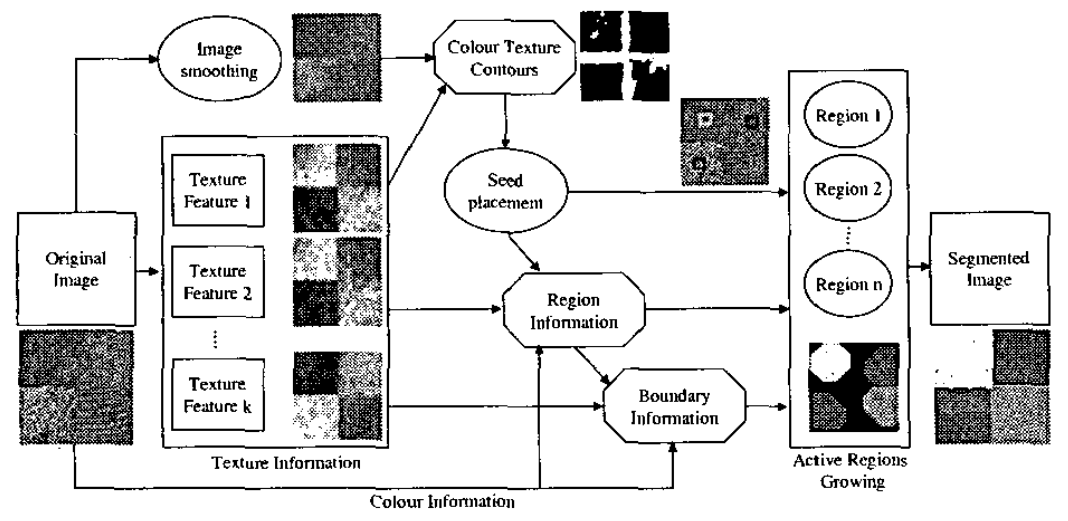

Fig. 1. Scheme of the proposed colour texture segmentation strategy.

tive regions. The knowledge of these regions allows to define the region information and to extract accurate boundary information. Then, as these regions grow, they compete for the pixels of the image by using a decision criterion which ensures the homogeneity inside the region and the presence of edges at its boundary. A scheme of the proposed strategy is shown in Fig. 1. The inclusion of colour information into our initial texture segmentation proposal involves two major issues: 1) the extraction of perceptual edges, 2) the modelling of colour and texture of regions.

\subsection{Initialisation: Perceptual Edges}

To obtain a sample of each region large enough to statistically model its behaviour, initial seeds have to be placed completely inside the regions. Boundary information allows us to extract these positions in the "core" of regions by looking for places far away from contours.

Boundaries between colour texture regions, which are combination of colour and texture edges, can be considered as perceptual edges because a human has the ability to detect both ones. The problem of texture edge detection is considered as a classical edge detection scheme in the multidimensional set of $k$ texture features which are used to represent the region characteristics. Meanwhile, the extraction of colour boundaries implies a major difficulty since the use of an edge detector over a colour image produces the apparition of microedges inside a textured region. Our approach is based on the perception of textures as homogeneous colour regions when they are seen from a long distance [6]. Hence, a smoothing process is progressively performed starting from the original image until textures looks homogeneous, as we would look the texture from far away. Then, the application of an edge detector allows to obtain the colour edges.
The union of texture and colour edges provides the perceptual edges of the image. Nevertheless, due to the inherently non-local property of texture and the smoothing process, the result of this method are inaccurate and thick contours. However, this information is enough to perform the seed placement in the "core" of regions, which allows to model the characteristics of regions.

\subsection{Colour Texture Region Information}

Colour in a textured region is by definition not homogenous and has a very variable behaviour between different regions of the image. Hence, methods which implicitly assume the same shape for all the clusters in the space, are not able to handle the complexity of the real feature space [7]. Therefore, we focus our attention on density estimation from a non-parametric approach since these methods do not have embedded assumptions, and specifically we adopt the kernel estimation technique. Considering colour pixels inside seeds as a set of data points assumed to be a sample of region colour, density estimation techniques allow the construction of an estimate of the probability density function which describes the behaviour of colour in a region. Given $n$ data points $x_{i}, i=1, \ldots, n$ in the d-dimensional space $R^{d}$, the multivariate kernel density estimator with kernel $K_{H}(x)$ and a bandwidth parameter $h$, becomes the expression

$$
\hat{f}(x)=\frac{1}{n h^{d}} \sum_{i=1}^{n} K_{H}\left(\frac{x-x_{i}}{h}\right)
$$

which gives us the probability of a pixel to belong to a region considering colour properties, $P_{R_{c}}$, on the threedimensional colour space. Note that in order to use only one bandwidth parameter $h>0$ the metric of the feature space has to be Euclidean. 
Texture of each region $R_{i}$ is modeled by a multivariate Gaussian distribution, so the mean vector $\overrightarrow{\mu_{i}}$ and the covariance matrix $\Sigma_{i}$, which are initialised from the seeds, describe the texture homogeneity region behaviour. Therefore, the probability of a pixel of belonging to a region considering textural properties, $P_{R_{t}}$, is given by the probability density function of a multivariate Gaussian distribution. Considering both properties together, the probability of a pixel $j$ of belonging to a region $R_{i}$ will be obtained considering the similarity of the colour pixel with the colour of the region, and the similarity of the texture around the pixel with the texture of the region. The combination of both terms gives the equation

$$
P_{R}\left(j \mid R_{i}\right)=\beta P_{R_{c}}\left(j \mid R_{i}\right)+(1-\beta) P_{R_{t}}\left(j \mid R_{i}\right)
$$

where $\beta$ weights the relative importance of colour and texture terms to evaluate the region information. The background is treated as a single region having uniform probability distribution $P_{0}$.

\subsection{Colour Texture Boundary Information}

It is well know that the extraction of boundary information for textured images is a very tougher task. Moreover, texture model of the adjacent textures is required to enable precise localization. The previous initialization step of the regions model allows to dispose of this required knowledge.

We shall consider that a pixel $j$ constitutes a boundary between two adjacent regions, $A$ and $B$, when the properties at both sides of the pixel are different and fit with the models of both regions. Textural and colour features are computed at both sides (referred as $m$ and its opposite as $n$ ). Therefore, $P_{R}(m \mid A)$ is the probability that features obtained in the side $m$ belong to region $A$, while $P_{R}(n \mid B)$ is the probability that the side $n$ corresponds to region $B$. Hence, the probability that the considered pixel is boundary between $A$ and $B$ is equal to $P_{R}(m \mid A) \times P_{R}(n \mid B)$, which is maximum when $j$ is exactly the edge between textures $A$ and $B$ because textures at both sides fit better with both models.

Four possible neighbourhood partitions (vertical, horizontal and two diagonals) are considered as in the proposal of Paragios and Deriche [2]. Therefore, the corresponding probability of a pixel $j$ to be boundary, $P_{B}(j)$, is the maximum probability obtained on the four possible partitions.

\subsection{Active Region Growing}

With the aim of integrating region and boundary information in an optimal segmentation, the global energy is defined with two basic terms. Boundary term measures the probability that boundary pixels are really edge pixels. Meanwhile, region term measures the homogeneity in the interior
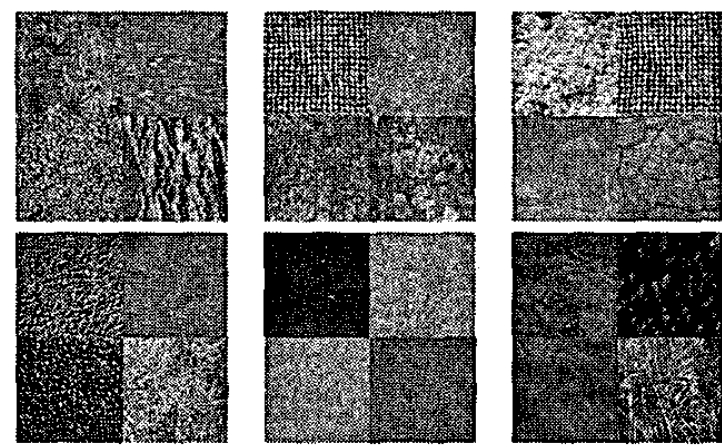

Fig. 2. Subset of mosaic colour texture images.

of the regions by the probability that these pixels belong to each corresponding region.

Some complementary definitions are required: let $\rho(R)=$ $\left\{R_{i}: i \epsilon[0, N]\right\}$ be a partition of the image into $\{N+1\}$ nonoverlapping regions, where $R_{0}$ is the region corresponding to the background region. Let $\partial \rho(R)=\left\{\partial R_{i}: i \epsilon[1, N]\right\}$ be the region boundaries of the partition $\rho(R)$. The energy function is then defined as

$$
\begin{aligned}
E(\rho(R))= & \left.(1-\alpha) \sum_{i=1}^{N}-\log P_{B}\left(j: j \epsilon \partial R_{i}\right)\right) \\
& +\alpha \sum_{i=0}^{N}-\log P_{R}\left(j: j \epsilon R_{i} \mid R_{i}\right)
\end{aligned}
$$

where $\alpha$ is a model parameter weighing both terms: boundary and region. This function is then optimized by a region competition algorithm [8] which takes the neighbouring pixels to the current region boundaries $\partial \rho(R)$ into account to determine the next movement. Specifically, a region aggregates a neighbouring pixel when this new classification decreases the energy of the segmentation. Intuitively, all regions begin to move and grow, competing for the pixels of the image until an energy minimum is reached. When the optimization process finishes, if there is a background region $R_{0}$ which remains without being segmented, a new seed is placed in the background, and the energy minimization starts again.

\section{EXPERIMENTAL RESULTS}

In order to evaluate the proposed colour texture segmentation technique, we created nine mosaic images by assembling four subimages of size $128 \times 128$ of textures from the VisTex natural scene collection by MIT (http://wwwwhite.media.mit.edu/vismod/imagery/VisionTexture/

/vistex html). Furthermore, we added three mosaics $M 10$, $M 11$ and $M 12$, provided by Dubuisson-Jolly and Gupta [9]. A subset of colour texture mosaic images is shown in Fig. 2. 
For the experimental trials shown in this paper we used the co-occurrence matrices, considering a window of size $7 \times 7$ centred at the analysed pixel. Two of the most typical features, contrast and homogeneity, are computed for distance one and for $0^{\circ}, 45^{\circ}, 90^{\circ}$ and $135^{\circ}$ orientations to constitute a 8-dimensional feature vector. Moreover, the Luv colour space has been chosen to model the colour.

The evaluation of image segmentation is performed by measuring the percentage of not-correctly segmented pixels considering the segmentation as a multi-class classification problem. Images were processed by our segmentation algorithm using various set of parameter values for the weight of colour (parameter $\beta$ ) and texture information, as well as the relative relevance of region (parameter $\alpha$ ) and boundary information in the segmentation process, and best results have been obtained with $\beta=0.6$ and $\alpha=0.75$. Note that a predominant role is given to colour and region information. Summarizing, an error mean of $2.218 \%$ has been obtained for the whole set of test images. Furthermore, our proposal obtained errors of $0.095 \%, 3.550 \%$ and $1.955 \%$ in the segmentation of $M 10, M 11$ and $M 12$, respectively (see segmentation results of $M 11$ and $M 12$ in Fig. 3). These results have to be considered as very positive since they improve colour texture segmentation results presented in [9].

\section{CONCLUSIONS}

A colour texture image segmentation method which integrates region and boundary information has been described. The algorithm uses the contours of the image in order to initialize a set of active regions. Therefore, colour texture of regions is modelled by the conjunction of non-parametric techniques of kernel density estimation and classical texture features. Then, region compete for the pixels optimizing an energy function which takes both region and boundary information into account.

The method has been evaluated on a set of mosaic images and experimental results corroborate conclusions from psychophysical studies which note the importance of colour on the human perception of colour texture [3]. Furthermore, obtained results demonstrate the effectiveness of the proposed algorithm.

\section{ACKNOWLEDGMENTS}

We would like to thank M.-P. Dubuisson-Jolly and A. Gupta at Imaging and Visualization Department, Siemens Corporate Research, for providing the texture mosaic images.

\section{REFERENCES}

[1] X. Muñoz, J. Freixenet, X. Cufí, and J. Martí, "Strategies for image segmentation combining region and

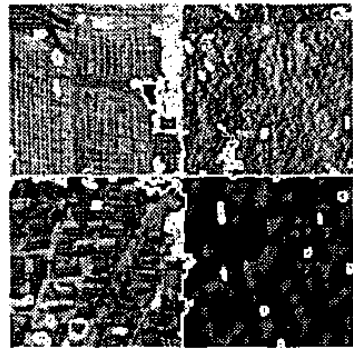

M11

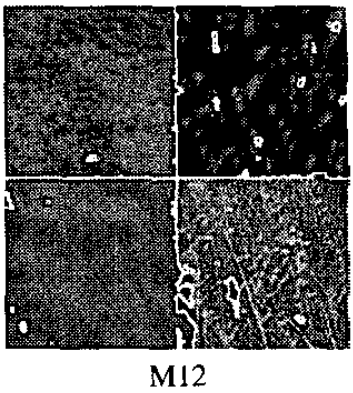

Fig. 3. Colour texture mosaic segmentation results. Borders of segmented regions are drawn over original images.

boundary information," Pattern Recognition Letters, vol. 24, no. 1-3, pp. 375-392, January 2003.

[2] N. Paragios and R. Deriche, "Geodesic active regions and level set methods for supervised texture segmentation," International Journal of Computer Vision, vol. 46 , no. 3, pp. 223-247, 2002.

[3] G.D. Finlayson and G.Y. Tian, "Investigating colour texture similarity," in Workshop on Texture Analysis in Machine Vision, Oulu, Finland, June 1999, pp. $127-$ 136.

[4] A. Drimbarean and P.F. Whelan, "Experiments in colour texture analysis," Pattern Recognition Letters, vol. 22, pp. 1161-1167, 2001.

[5] X. Muñoz, J. Freixenet, J. Martí, and X. Cufí, “Active regions for unsupervised texture segmentation integrating region and boundary information," in International Workshop on Texture Analysis and Synthesis, Copenhagen, Denmark, June 2002, pp. 95-98.

[6] M. Mirmehdi and M. Petrou, "Segmentation of color textures," IEEE PAMI, vol. 22, no. 2, pp. 142-159, February 2000.

[7] D. Comaniciu and P. Meer, "Mean shift: A robust approach toward feature space analysis," IEEE PAMI, vol. 24, no. 5, pp. 1-18, April 2002.

[8] S.C. Zhu and A. Yuille, "Region competition: Unifying snakes, region growing, and bayes/mdl for multi-band image segmentation," IEEE PAMI, vol. 18, no. 9, pp. 884-900, September 1996.

[9] M.-P. Dubuisson-Jolly and A. Gupta, "Color and texture fusion: Application to aerial image segmentation and gis updating," Image and Vision Computing, vol. 18 , pp. 823-832, 2000. 